\title{
Deeply virtual Compton scattering at the EIC and LHeC: a comparison among saturation approaches
}

\author{
D. Bendova ${ }^{1, a}$, J. Cepila ${ }^{1, b}$, V. P. Gonçalves ${ }^{2, c}$, C. R. Sena ${ }^{2}$ \\ ${ }^{1}$ Faculty of Nuclear Sciences and Physical Engineering, Czech Technical University in Prague, Břehová 7, 11519 Prague, Czech Republic \\ ${ }^{2}$ High and Medium Energy Group, Instituto de Física e Matemática, Universidade Federal de Pelotas (UFPel), Caixa Postal 354, Pelotas, RS \\ 96010-900, Brazil
}

Received: 13 November 2021 / Accepted: 25 January 2022 / Published online: 1 February 2022

(C) The Author(s) 2022

\begin{abstract}
The impact of nonlinear effects in the deeply virtual Compton scattering (DVCS) process that will be measured in future electron-hadron collisions is investigated. We present, for the first time, the predictions derived using the solution to the Balitsky-Kovchegov equation with the collinearly-improved kernel and including the impactparameter dependence. We estimate the total cross section and $t$-distribution of the DVCS process in $e p$ and $e A$ collisions and demonstrate that $d \sigma / d t$ is strongly dependent on the assumption for the impact-parameter dependence of the dipole-hadron scattering amplitude. Our results indicate that a future experimental analysis of this process will be useful to discriminate among different models for the saturation physics and, consequently, will allow us to constrain the description of QCD dynamics in parton densities.
\end{abstract}

\section{Introduction}

One of the main goals of the future electron-ion colliders at BNL (EIC) [1-3] and CERN ( $\mathrm{LHeC}$ ) [4,5] is to improve our understanding about the quantum 3D imaging of the partons inside the protons and nuclei by measuring the parton position, momentum, and angular momentum with unprecedent precision. Theoretically, all quantum information of how partons are distributed inside hadrons is encoded in the quantum phase space Wigner distributions, which include information on both generalized parton distributions (GPDs) and transverse momentum parton distributions (TMDs), see e.g. Refs. [6-13]. Experimentally, the tomography picture from the hadrons can be revealed in deep inelastic electronhadron scattering by measurements of exclusive processes,

\footnotetext{
a e-mail: dagmar.bendova@fjfi.cvut.cz

b e-mail: jan.cepila@fjfi.cvut.cz

c e-mail: barros@ufpel.edu.br (corresponding author)
}

wherein the hadron remains intact after scattering of the lepton probe. One has that the Fourier transform of the associated differential cross sections $d \sigma / d t$, where $t$ is the squared four-momentum transfer between the incoming and scattered hadron, can be used to obtain the transverse spatial distributions of quarks and gluons in both protons and nuclei. In particular, the future experimental analysis of the diffractive dijet production [14-19] and the deeply virtual Compton scattering (DVCS) [20-22] at the EIC and $\mathrm{LHeC}$ are expected to directly probe the gluon GPD.

In this paper, we will focus on coherent DVCS processes represented in the Fig. 1, i.e. production of a real photon with the target hadron remaining intact. ${ }^{1}$ At large energies, this process is driven by the gluon content of the target, with the cross section being proportional to the square of the scattering amplitude and, consequently, being strongly sensitive to the underlying QCD dynamics. For previous studies, see e.g. Refs. [23-28]. Moreover, the behaviour of $d \sigma / d t$ at small- $x$ is determined by the impact-parameter dependence of the scattering amplitude $\mathcal{N}\left(x, \boldsymbol{r}, \boldsymbol{b}_{t}\right)$ of a dipole off the target, where $\boldsymbol{r}$ is the transverse dipole size and $\boldsymbol{b}_{t}$ the impact - parameter. The amplitude encodes the information about the hadronic scattering as well as the nonlinear and quantum effects in the hadron wave function [29-31]. In recent years, several groups have proposed different phenomenological approaches to describe $\mathcal{N}\left(x, \boldsymbol{r}, \boldsymbol{b}_{t}\right)$, which are based on the color glass condensate (CGC) formalism [32-37] and successfully describe a large set of observables in $e p, p p, p A$, and $A A$ collisions. In particular, the IP-Sat $[25,38]$ and b-CGC $[39,40]$ approaches are able to describe the DVCS data obtained in the kinematical range covered

\footnotetext{
1 The same final state is also generated by the Bethe-Heitler $(\mathrm{BH})$ process, where the elastic electron-hadron scattering is followed by photon emission off the electron. In our analysis, we will assume that the DVCS contribution can be experimentally separated as was done e.g. at HERA.
} 


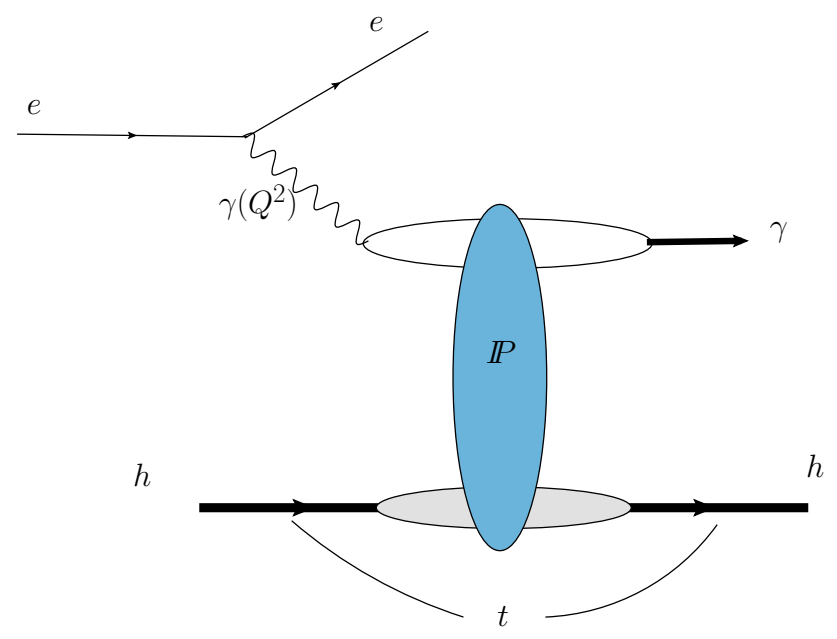

Fig. 1 Deeply virtual Compton scattering in coherent diffractive processes, where the hadron can be a proton or a nucleus

by the HERA experiment. A shortcoming of these models is that the associated $\boldsymbol{b}_{t}$ dependencies are not based on the solutions of the Balitsky-Kovchegov (BK) equation [41-45], which is the mean-field approximation of the infinite hierarchy of coupled equations for the correlators of Wilson lines predicted by the CGC formalism [32-37,41-43]. Moreover, these models have been proposed for a proton target and its extension for a nuclear target is, in general, performed using the Glauber-Gribov (GG) approach [46-49] (see e.g. Refs. [51-53]). Recently, in Refs. [54-56], the impact-parameter and energy dependencies of $\mathcal{N}\left(x, \boldsymbol{r}, \boldsymbol{b}_{t}\right)$ have been derived by solving the BK equation and taking into account the collinear corrections [57-59] to the kernel of the evolution equation for both proton and nuclear targets. One of the goals of this paper is to estimate the DVCS cross sections using this approach and present, for the first time, a comparison between its predictions and the HERA data. Another goal is to present an extensive comparison between the predictions of the distinct saturation approaches for the energy dependence of the total cross section and for the $t$-distributions considering the kinematical range covered by the future electron-ion colliders. Our main motivation is to verify if future experimental data for the DVCS process could be used to discriminate between these distinct treatments of the QCD dynamics at high energies.

The paper is organized as follows. In the next section, we present a brief overview of the color dipole formalism for the description of deeply virtual Compton scattering and the distinct models for the dipole-target scattering amplitude used in our analysis. In Sect.3, we present our predictions for the total cross section and the $t$-distribution considering $e p$ and $e A$ collisions. Finally, in Sect. 4, we summarize our main conclusions.

\section{Formalism}

Let us start presenting a brief review of the deeply virtual Compton scattering (DVCS) in electron-hadron collisions, represented in Fig. 1. This process is characterized by a real photon and an intact hadron in the final state, with a rapidity gap separating these systems. In the color dipole approach the scattering amplitude for the exclusive real photon production $\gamma^{*} h \rightarrow \gamma h$ can be factorized in terms of the fluctuation of the virtual photon into a $q \bar{q}$ color dipole, the dipole-hadron scattering by a color singlet exchange (IP) and the recombination into the exclusive final state $\gamma$. As demonstrated e.g. in Refs. [21,22,25], the DVCS amplitude can be written as ${ }^{2}$

$$
\begin{aligned}
& A^{\gamma^{*} h \rightarrow \gamma h}\left(x, r, \Delta, Q^{2}\right)=\int d b_{t}\left(2 \pi b_{t}\right) \int d r(2 \pi r) \\
& \quad \times \int d z \sum_{f}\left(\Psi_{\gamma *}^{*} \Psi_{\gamma}\right)^{f} J_{0}\left(b_{t} \Delta\right) J_{0}([1-2 z] r \Delta / 2) \frac{d \sigma_{q \bar{q}}}{d^{2} \boldsymbol{b}_{t}}
\end{aligned}
$$

where $r$ is the size of the $q \bar{q}$ dipole, $\boldsymbol{b}_{t}$ is the impact - parameter, $\Delta^{2}=-t$, and $z$ and $(1-z)$ are the momentum fractions of the incoming photon carried by the quark and anti-quark, respectively. Moreover, the overlap function $\left(\Psi_{\gamma *}^{*} \Psi_{\gamma}\right)^{f}$ is given by

$$
\begin{aligned}
\left(\Psi_{\gamma *}^{*} \Psi_{\gamma}\right)^{f}= & \frac{N_{c} \alpha_{e m}}{2 \pi^{2}} e_{f}^{2} \\
& \times\left\{\left[z^{2}+(1-z)^{2}\right] \epsilon_{1} K_{1}\left(\epsilon_{1} r\right) \epsilon_{2} K_{1}\left(\epsilon_{2} r\right)\right. \\
& \left.+m_{f}^{2} K_{0}\left(\epsilon_{1} r\right) K_{0}\left(\epsilon_{2} r\right)\right\},
\end{aligned}
$$

where $m_{f}$ and $e_{f}$ are the mass and the charge of a quark with flavor $f$. The cross section for the dipole scattering off the target at an impact - parameter $\boldsymbol{b}_{t}$ is denoted by $\mathrm{d} \sigma_{q \bar{q}} / \mathrm{d}^{2} \boldsymbol{b}_{t}$ and it is related to the dipole-target scattering amplitude $\mathcal{N}_{h}\left(x, \boldsymbol{r}, \boldsymbol{b}_{t}\right)$ by

$\frac{\mathrm{d} \sigma_{q \bar{q}}}{\mathrm{~d}^{2} \boldsymbol{b}_{t}}=2 \mathcal{N}_{h}\left(\bar{x}, \boldsymbol{r}, \boldsymbol{b}_{t}\right)$,

where $\bar{x}=x\left(1+4 m_{f}^{2} / Q^{2}\right)$, with $x$ being the Bjorken variable. As a consequence, one has that the energy, photon virtuality, transverse momentum, and atomic number dependencies of DVCS cross section are determined by the evolution of $\mathcal{N}$ and, consequently, they strongly depend on the description of the QCD dynamics. An important aspect, that must be emphasized, is that although the DVCS cross section is smaller than the vector meson one, it is not affected by the theoretical uncertainties associated to the scarce knowledge

\footnotetext{
2 In our analysis we will disregard the correlations between $\boldsymbol{r}$ and $\boldsymbol{b}_{t}$ in the dipole-target scattering amplitude, which is a good approximation for the calculation of the total cross section and the $t$-distribution. However, less inclusive observables, as e.g. the electron-photon azimuthal angle correlation, are sensitive to nontrivial spatial correlations in the gluon distribution of the target [22].
} 


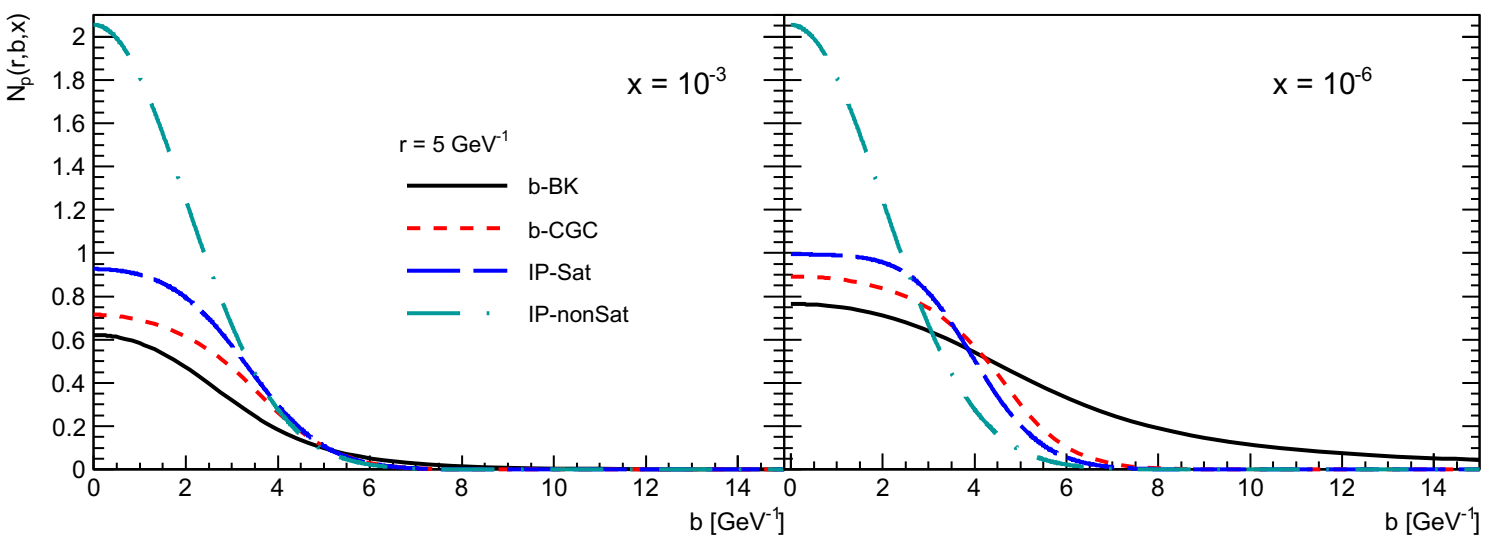

Fig. 2 Predictions of the different nonlinear approaches for the impact-parameter dependence of the dipole-proton scattering amplitude considering two distinct values of $x\left(=10^{-3}\right.$ and $\left.10^{-6}\right)$ and a fixed dipole size $\left(r=5 \mathrm{GeV}^{-1}\right)$

of the vector meson wave functions. Which implies that the DVCS process can be considered a direct probe of the QCD dynamics for the dipole-target interaction.

In the CGC formalism [32-37], the scattering amplitude can be obtained by solving the BK equation, which is given by [41-45]

$$
\begin{aligned}
\frac{\partial \mathcal{N}\left(\boldsymbol{r}, \boldsymbol{b}_{t}, Y\right)}{\partial Y}= & \int \mathrm{d} \boldsymbol{r}_{1} K\left(\boldsymbol{r}, \boldsymbol{r}_{1}, \boldsymbol{r}_{2}\right)\left[\mathcal{N}\left(\boldsymbol{r}_{1}, \boldsymbol{b}_{1}, Y\right)\right. \\
& +\mathcal{N}\left(\boldsymbol{r}_{2}, \boldsymbol{b}_{2}, Y\right)-\mathcal{N}\left(\boldsymbol{r}, \boldsymbol{b}_{t}, Y\right) \\
& \left.-\mathcal{N}\left(\boldsymbol{r}_{1}, \boldsymbol{b}_{1}, Y\right) \mathcal{N}\left(\boldsymbol{r}_{2}, \boldsymbol{b}_{2}, Y\right)\right],
\end{aligned}
$$

where $Y$ is the rapidity, $r \equiv|\boldsymbol{r}|, r_{1} \equiv\left|\boldsymbol{r}_{1}\right|$ and $r_{2} \equiv\left|\boldsymbol{r}_{2}\right|$ are the transverse sizes of the original dipole and of the two daughter dipoles, respectively, and $b_{i} \equiv\left|\boldsymbol{b}_{i}\right|$ are the corresponding impact parameters. The evolution runs in rapidity $Y=\ln \left(x_{0} / x\right)$, where $x$ is the Bjorken variable and $x_{0}$ gives the initial value of Bjorken variable for the evolution. The form of the kernel, $K\left(\boldsymbol{r}, \boldsymbol{r}_{\mathbf{1}}, \boldsymbol{r}_{\mathbf{2}}\right)$, depends on the approximations assumed to treat the next-to-leading order corrections associated e.g. to the running of the coupling constant and collinear logarithms that arise in the perturbative expansion. As pointed out in the Introduction, we will focus in this study on the approach proposed in Refs. [54-56], which is based on the solution of the BalitskyKovchegov equation for the dipole-hadron scattering amplitude including the dependence on impact-parameter and using the collinearly-improved kernel proposed in Refs. [5759]. In such approach, the power-like (Coulomb) tail at large impact parameters, found in the solution of the BK equation at leading order in Ref. [60], is suppressed by higher-order corrections that impose time-ordering of the gluon emissions. In Refs. [54,55], the BK equation with the collinearlyimproved kernel was solved for a proton target assuming that the initial condition is given by a combination of the GBW model $[63,64]$ for the dependence on the dipole size $r$ and of a Gaussian distribution for the impact-parameter dependence. The parameters have been fixed using HERA data for $F_{2}$ and for the $|t|$-distribution of the $J / \Psi$ photoproduction. The resulting solutions will be denoted b-BK in what follows. In Fig. 2, we present the impact-parameter dependence of the b-BK solution for a proton target considering two values of $x$ and a fixed dipole size. Such predictions are compared with the predictions from the IP-Sat and b-CGC models (see e.g. Refs. $[25,38,40]$ ), which are phenomenological models based on the CGC physics that assume distinct ad hoc impact-parameter dependencies for the scattering amplitude. In addition, we also present the predictions of the IP-nonSat model, which can be derived from the IP-Sat model by disregarding the impact of the multiple scattering corrections that take into account of the nonlinear QCD effects in this model. Therefore, the comparison between the IP-nonSat predictions and those from the other models allow us to estimate the impact of the saturation effects for a proton target. For $x=10^{-3}$ (left panel), one has that predictions are similar for large impact - parameters. In contrast, the predictions for small- $b$ are distinct, with the IP-nonSat model violating the unitarity bound expressed by $\mathcal{N}_{p} \leq 1$. Such violation is also observed for smaller values of $x$ (right panel). Moreover, the distributions predicted by the b-BK, IP-Sat and b-CGC models become wider with the decreasing of $x$, where the behaviour of $\mathcal{N}_{p}$ at large- $b$ depends on the dipole model considered. In particular, the b-BK solution predicts a long tail, as already demonstrated in Refs. [54,55]. Such results motivate the study of observables that are sensitive to the impact-parameter dependence and, consequently, to the modelling of $\mathcal{N}$. The $t$-distributions represent a useful tool here, since $t$ and $b$ are Fourier conjugated variables.

For the nuclear case, the DVCS amplitude can be estimated assuming the Glauber-Gribov (GG) formalism [4650], as performed e.g. in Refs. [26,28]. In this approach, the dipole-nucleus scattering amplitude $\mathcal{N}_{A}\left(\boldsymbol{r}, \boldsymbol{b}_{A}, Y\right)$ is given 
Fig. 3 Predictions of the different nonlinear approaches for the impact-parameter dependence of the dipole-nucleus scattering amplitude considering two distinct values of $x\left(=10^{-3}\right.$ and $\left.10^{-6}\right)$, a fixed dipole size $(r=5$ $\left.\mathrm{GeV}^{-1}\right)$, and $A=P b$

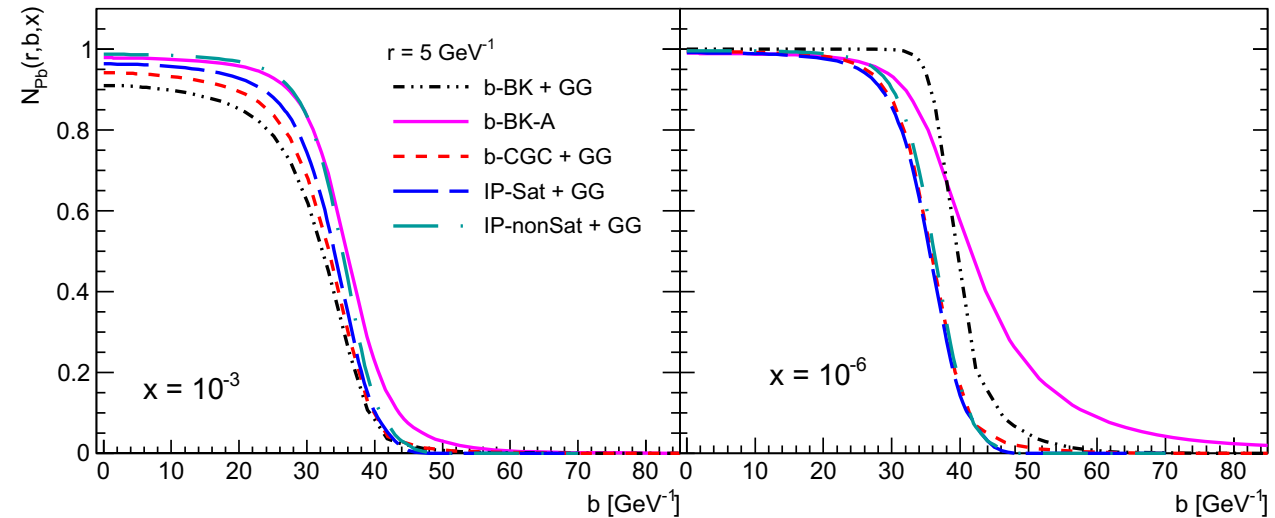

by

$\mathcal{N}_{A}\left(\boldsymbol{r}, \boldsymbol{b}_{A}, Y\right)=1-\exp \left[-\frac{1}{2} \sigma_{d p}\left(Y, \boldsymbol{r}^{2}\right) T_{A}\left(\boldsymbol{b}_{A}\right)\right]$,

where $\boldsymbol{b}_{A}$ is the dipole-nucleus impact-parameter and the nuclear profile function $T_{A}\left(\boldsymbol{b}_{A}\right)$ is described by a WoodsSaxon distribution. The dipole-proton cross section, denoted as $\sigma_{d p}$, is expressed in terms of the dipole-proton scattering amplitude as follows

$\sigma_{d p}\left(Y, \boldsymbol{r}^{2}\right)=2 \int \mathrm{d}^{2} \boldsymbol{b}_{p} \mathcal{N}_{p}\left(\boldsymbol{r}, \boldsymbol{b}_{p}, Y\right)$,

with $\boldsymbol{b}_{p}$ being the impact-parameter for the dipole-proton interaction. In this approach, the IP-Sat, IP-nonSat, b-CGC and $\mathrm{b}$-BK models for a proton target can be used as input to estimate $\mathcal{N}_{A}\left(\boldsymbol{r}, \boldsymbol{b}_{A}, Y\right)$. In what follows, the associated predictions will be denoted as IP-Sat + GG, IP-nonSat + GG, b-CGC + GG and b-BK + GG, respectively. In contrast, $\mathcal{N}_{A}$ can be directly estimated by solving the BK equation for the nuclear case, following the approach proposed in Refs. $[54,55]$. In particular, in Ref. [56], the authors extended the approach for a nuclear target assuming that the initial condition is given by

$$
\begin{aligned}
& \mathcal{N}_{A}\left(\boldsymbol{r}, \boldsymbol{b}_{A}, Y=0\right) \\
& \quad=1-\exp \left[-\frac{1}{2} \frac{Q_{s 0}^{2}(A)}{4} r^{2} T_{A}\left(\boldsymbol{b}_{q_{1}}, \boldsymbol{b}_{q_{2}}\right)\right],
\end{aligned}
$$

where $Y=\ln \left(x_{0} / x\right)$ with $x_{0}=0.008, \boldsymbol{b}_{q_{i}}$ are the impact - parameters with respect to the dipole constituents, and $Q_{s 0}^{2}$ is a free parameter determined for each value of $A$ by the comparison between the dipole predictions for $F_{2}^{A}$ and those obtained using the collinear formalism and the EPPS16 parametrization [61] for $Y=0$. Moreover, $T_{A}\left(\boldsymbol{b}_{q_{1}}, \boldsymbol{b}_{q_{2}}\right)$ is the nuclear profile, which is assumed to be given by

$T_{A}\left(\boldsymbol{b}_{q_{1}}, \boldsymbol{b}_{q_{2}}\right)=k\left[T_{A}\left(\boldsymbol{b}_{q_{1}}\right)+T_{A}\left(\boldsymbol{b}_{q_{2}}\right)\right]$,

where the individual profiles $T_{A}\left(\boldsymbol{b}_{q_{i}}\right)$ are described by a Woods-Saxon distribution and $k$ is the factor which ensures $k T_{A}(0)=1$ and implies that Eq. (7) becomes the GBW formula $[63,64]$ for $\boldsymbol{b}=0$. The associated predictions will be denoted as b-BK-A hereafter. In Fig. 3, we compare the predictions of the different approaches for the impact-parameter dependence of $\mathcal{N}_{A}$ considering two values of $x$, a fixed dipole size, and $A=P b$. One has that all models predict $\mathcal{N}_{A} \leq 1$, i.e., they all satisfy unitarity. However, the $b$-dependence is strongly model dependent. In particular, the behaviours predicted by the b-BK + GG and b-BK-A models for small- $x$ and large- $b$ are significantly distinct, with the b-BK-A predicting a longer tail. Such result motivates the analysis of the impact of these distinct descriptions of the nonlinear effects on the $t$-distributions of the nuclear DVCS process, which could be measured in future $e A$ colliders.

\section{Results}

In this section we will investigate the impact of the different treatments of the nonlinear effects on the total cross section and $t$-distributions considering the kinematical range that will be covered by the future electron-ion colliders EIC and LHeC [1-5]. These two observables are directly related, since the total cross section for the exclusive real photon production is given by

$$
\begin{aligned}
\sigma^{\gamma^{*} h \rightarrow \gamma h}\left(W, Q^{2}\right)= & \int_{-\infty}^{0} d t \frac{d \sigma^{\gamma^{*} h \rightarrow \gamma h}}{d t} \\
= & \int_{-\infty}^{0} d t \frac{1}{16 \pi} \mid A^{\gamma^{*} h \rightarrow \gamma h} \\
& \left.\left(x, r, \Delta, Q^{2}\right)\right|^{2}
\end{aligned}
$$

where $W$ is the photon-hadron center-of-mass energy, $Q^{2}$ is the virtuality of the incoming photon and the amplitude is given by Eq. (1). As in Ref. [25], the differential cross section for a proton target will be multiplied by the factor $R_{g}^{2}\left(1+\beta^{2}\right)$ in order to take into account the skewness effect $\left(R_{g}\right)$ and the real part of the scattering amplitude $(\beta)$. The skewness correction is related to the fact that in the twogluon exchange limit, the gluons emitted from the quark and antiquark into the dipole can carry different momentum frac- 

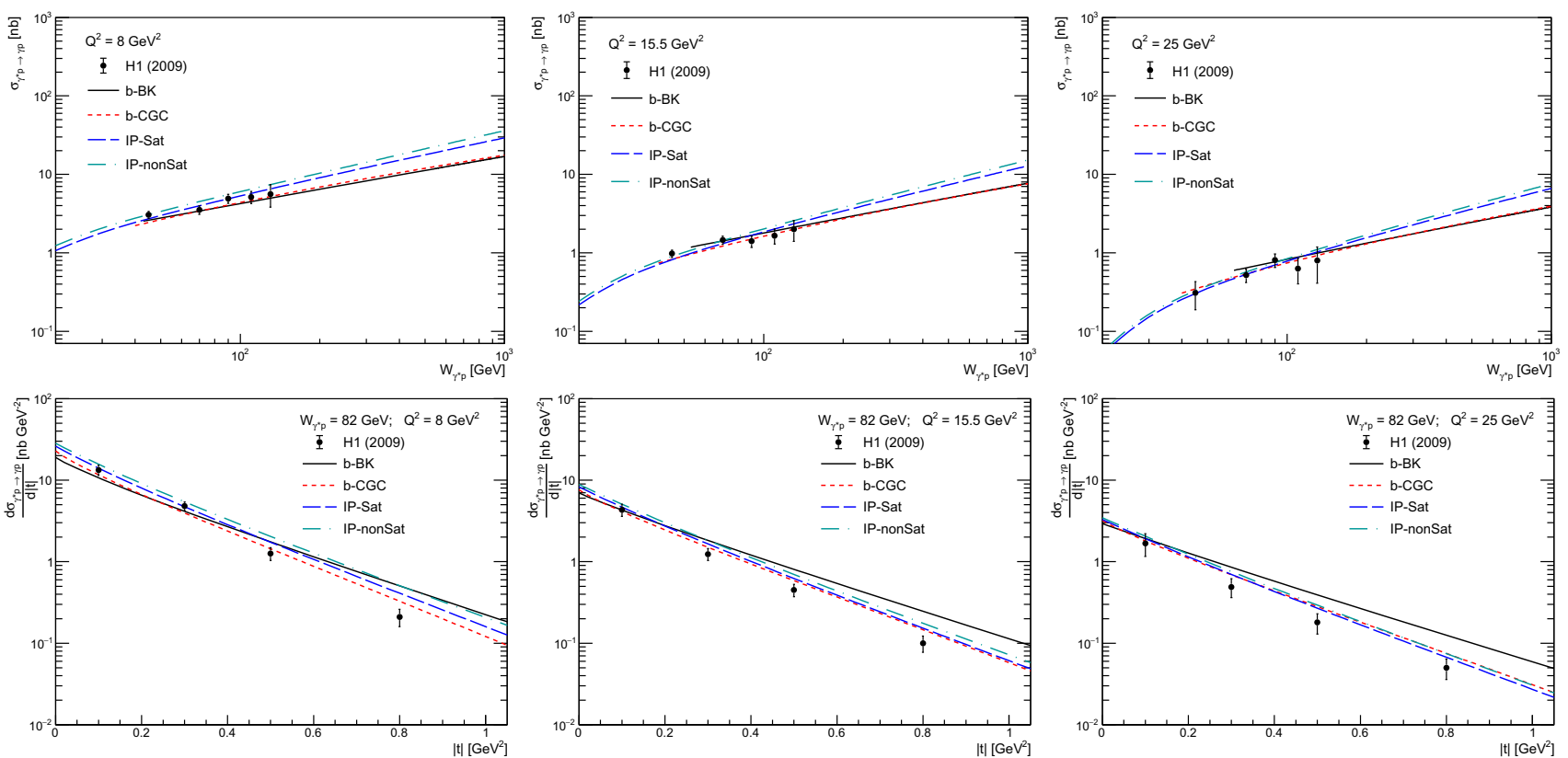

Fig. 4 Predictions for the energy dependence (upper panels) and $t$-distributions (lower panels) of the DVCS process in $e p$ collisions at HERA derived considering distinct models for the dipole-proton scattering amplitude and different values of the photon virtuality. Data from H1 [66]

tions. Such correction has been derived in the framework of collinear factorization [65], and its application in the dipole approach is still under debate. However, the comparison of the dipole predictions with the HERA data indicate that the skewness and real part corrections are needed to describe the data. In our analysis, we will assume $R_{g}^{2}=1.1$ and $\beta=0.3$ for all models considered, which is a good first approximation for the value calculated considering the distinct dipole models. In contrast, for a nuclear target, we will disregard these corrections, since the calculation of $R_{g}$ and $\beta$ for the nuclear case is a subject of still ongoing discussions. The IP-Sat and IP-nonSat predictions will be derived using the parameters obtained in Ref. [62]. For the b-CGC model, we consider the parameters adjusted to the latest HERA data in Ref. [40].

Initially, in Fig. 4, we present the predictions for the energy dependence of the total cross section (upper panels) and the cross section $|t|$-distribution (lower panels) assuming different values for the photon virtuality and considering the b-CGC, IP-Sat, IP-nonSat and b-BK models for the dipoleproton scattering amplitude. The data from $\mathrm{H} 1$ are presented for a comparison [66]. One has that the HERA data for the energy dependence of the total cross section is well described by all models, with the b-BK and b-CGC predictions being similar. In contrast, the IP-sat and IP-nonSat predictions are steeper in energy, with the IP-nonSat predictions being $20 \%$ larger than the IP-Sat one for large energies. Our results indicate that the difference between the predictions increase with energy and with the decreasing of the photon virtuality. Such results are expected, since the impact of the saturation effects is larger for small values of $x$ and/or $Q^{2}$. In the lower panels of Fig. 4, we present the results for $d \sigma / d t$, which indicate that the large- $t$ behaviour of the differential distribution is sensitive to dipole model considered. In particular, the b-BK model predicts a milder decrease with $t$ in the kinematical range probed by HERA. This result seems to disfavor the b-BK model with respect to the other CGC-inspired models, with the discrepancy growing with increasing $Q^{2}$. This may suggest that effects related to the $Q^{2}$ evolution starts to play a role and that the model based on the b-BK solutions doesn't include these effects to a necessary extent. However, since the total cross section seems to be well described and the model provided good description of other processes in previous studies (see e.g. $[54,55,67]$ ), we have decided not to disregard this model in the subsequent predictions for future experiments which may come with new results with respect to the previous measurements.

Exclusive events characterized by larger values of $|t|$ than those observed at HERA are expected to be measured in future $e p$ colliders. That will allow us to probe the presence and position of dips predicted to occur in diffractive processes when nonlinear effects are taken into account. In Fig. 5, we present our predictions for the $t$-distribution of the DVCS process in $e p$ collisions at the EIC (upper panels) and LHeC (lower panels) considering distinct models for $\mathcal{N}_{p}$. The results indicate that the distribution strongly depends on the model considered. In particular, the IP-nonSat model does not predict the presence of a dip in the $|t|$-distribution. In contrast, the models based on the CGC physics predict dips at large values of $|t|$, with its positions being dependent 

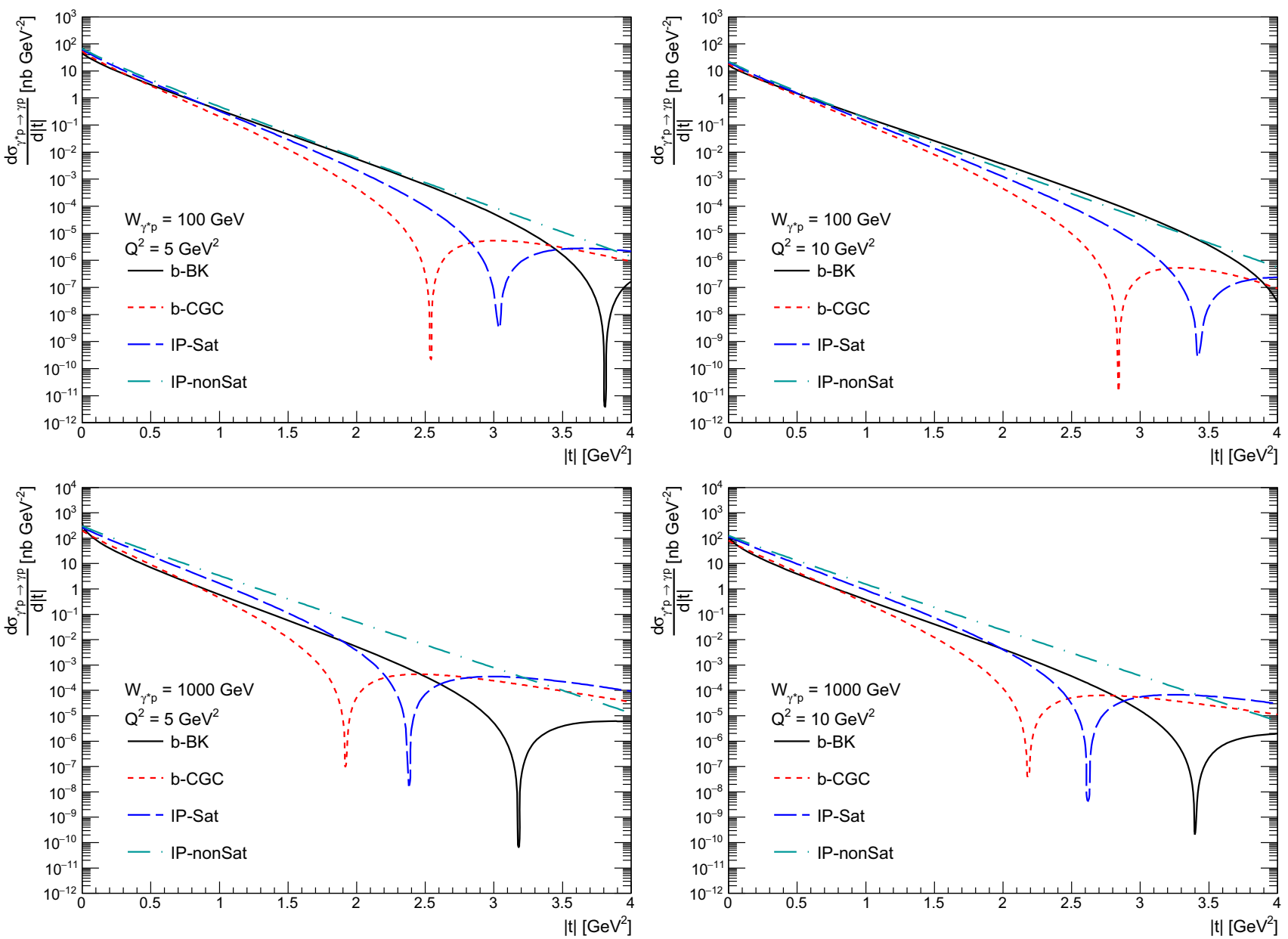

Fig. 5 Predictions for the $t$-distributions of the DVCS process in ep collisions at the EIC (upper panels) and LHeC (lower panels) derived considering distinct models for the dipole-proton scattering amplitude and different values of the photon virtuality
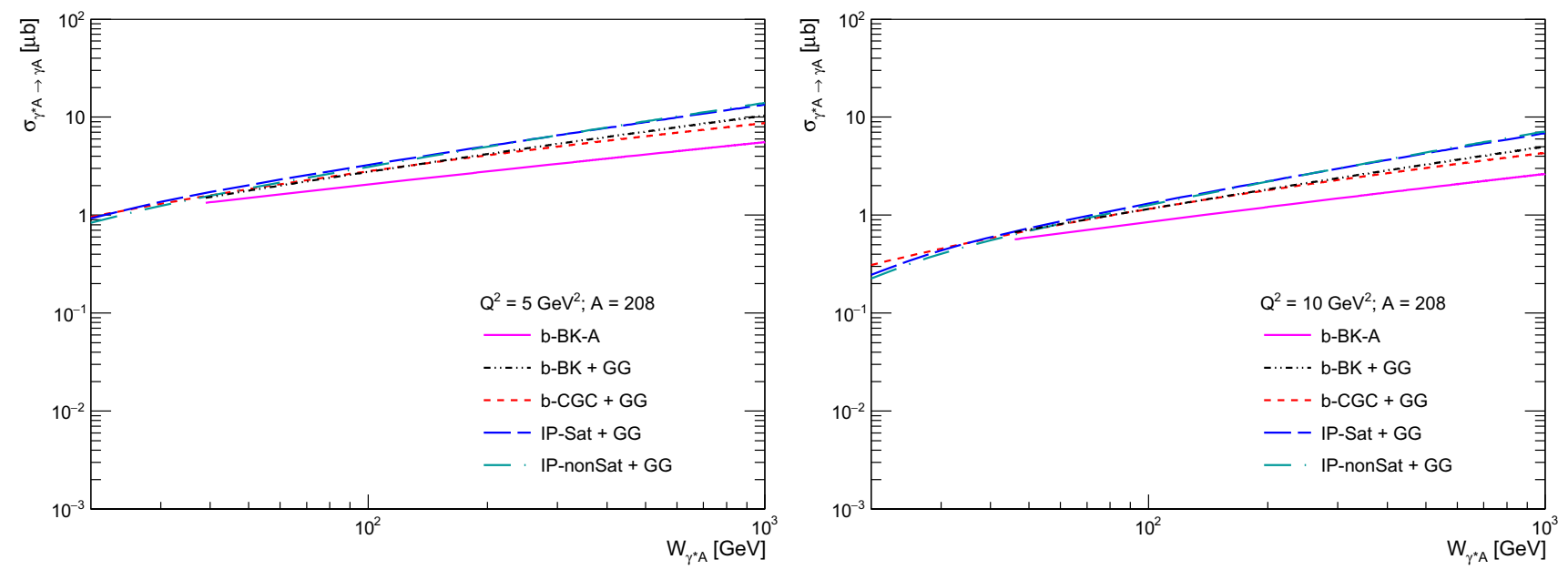

Fig. 6 Predictions for the energy dependence of the nuclear DVCS cross section considering distinct models for the dipole-nucleus scattering amplitude, $A=P b$, and different values of the photon virtuality 

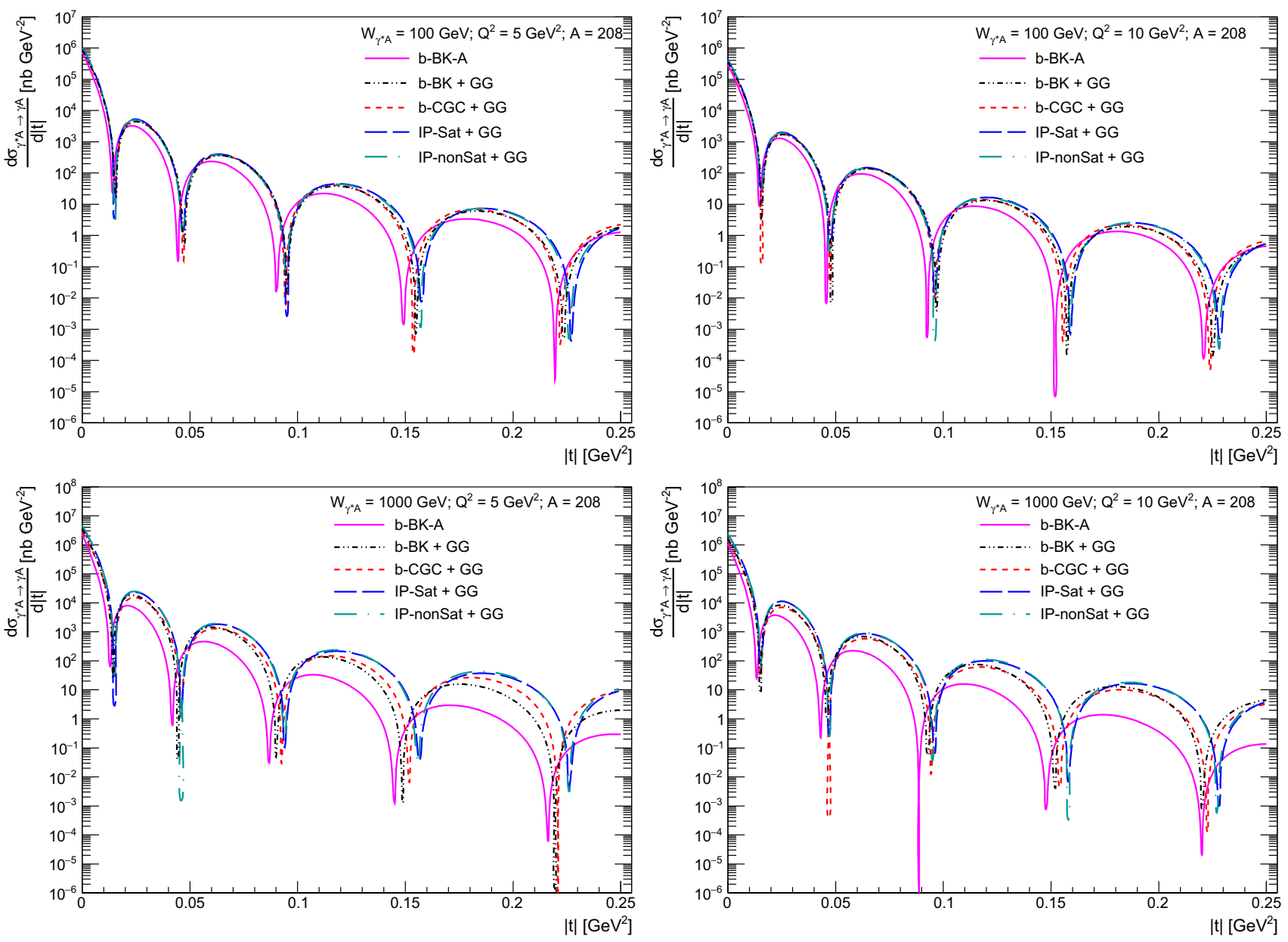

Fig. 7 Predictions for the $t$-distribution of the nuclear DVCS process at the EIC (upper panels) and LHeC (lower panels) derived considering distinct models for the dipole-nucleus scattering amplitude, $A=P b$, and different values of the photon virtuality

on the model considered. One has that the first dip occurs for smaller values of $|t|$ when the center-of-mass energy is increased and/or when a smaller value of the photon virtuality is assumed. The large difference in the position of the dips predicted by the distinct models implies that a future measurement could be able to discriminate between these different approaches for the QCD dynamics at high energies.

In what follows, we will consider the DVCS process in $e A$ collisions. Such analysis is motivated by the expectation that nuclei are an efficient amplifier of nonlinear effects [29-31]. The predictions for the energy dependence of the total DVCS cross section for a nuclear target $(A=P b)$ are presented in Fig. 6. One has that they are similar for small $W$ but can differ by a factor $\gtrsim 2$ at the $\mathrm{LHeC}$ energy scale. The IP-Sat + GG and IP-nonSat + GG predictions appear to be almost identical for the values of $Q^{2}$ considered. We have verified that they start to be different for smaller values of the photon virtuality, where the impact of the nonlinear effects at the proton level becomes more significant. Moreover, the b-CGC + GG and b$\mathrm{BK}+\mathrm{GG}$ predictions are similar even with increasing energy, while the b-BK-A one is the lowest of the presented models for the magnitude of the total cross section.

In Fig. 7, we present the predictions for the $t$-distributions of the nuclear DVCS process at the EIC (upper panels) and $\mathrm{LHeC}$ (lower panels) for two values of photon virtuality and $A=P b$, derived considering distinct models for the dipolenucleus scattering amplitude. One has that the position of the first two dips are similar for the predictions based on Glauber-Gribov approach and become gradually distinct at larger values of $|t|$. In other words, in order to discriminate the treatment of the QCD dynamics at the proton level, we should probe values of $|t| \gtrsim 0.1 \mathrm{GeV}^{2}$. In contrast, future data for smaller values of $|t|$ can be useful to discriminate between the GG and the b-BK-A approaches for the dipole-nucleus scattering amplitude. Our results indicate that the difference between the predictions increases with the center-of-mass energy, becoming appreciable in the $\mathrm{LHeC}$ kinematic range.

A comment is order. One has verified that similar results are obtained for $A=40$, with the main differences being the smaller normalizations, decrease of the difference between 
the predictions and the change in the position of the dips. These results indicate that the study of $e A$ collisions with lighter nuclei can also be useful, in particular due to the different atomic number dependence of the saturation scale predicted by the nonlinear approaches considered in our analysis.

\section{Summary}

The experimental analysis of the diffractive observables in future electron-hadron collisions at EIC and $\mathrm{LHeC}$ is expected to improve our understanding about the QCD dynamics at high parton densities and the multi-dimensional description of the partons within the hadrons. In particular, the study of the deeply virtual Compton scattering is predicted to be a probe of the gluon Wigner distribution. In this paper, we have investigated the impact of the distinct treatments for the nonlinear (saturation) effects on the DVCS process. We have presented, for the first time, the predictions derived using the solution of the impact-parameter dependent Balitsky-Kovchegov equation for the dipole-target scattering amplitude. We have presented these predictions for $e p$ and $e A$ collisions, considering the kinematic ranges that will be probed by the future EIC and LHeC colliders. A comprehensive comparison with the predictions derived using alternative saturation approaches was performed. Our results demonstrate that the cross section and $t$-distribution are strongly dependent on the assumption for the impactparameter dependence of the dipole-target scattering amplitude. As a consequence, we predict that the tomography picture of a hadron will be sensitive to the nonlinear effects. Such results indicate that a future experimental analysis of the DVCS process will be useful to improve our understanding of QCD dynamics and hadronic structure.

Acknowledgements VPG would like to thank the members of the Czech Technical University in Prague for the warm hospitality during the beginning of this project. VPG and CRS were partially financed by the Brazilian funding agencies CNPq, FAPERGS and INCTFNA (processes number 464898/2014-5). This work has been supported by the Centre of Advanced Applied Sciences with the number: CZ.02.1.01/0.0/0.0/16-019/0000778. The Centre of Advanced Applied Sciences is co-financed by the European Union.

Data Availability Statement This manuscript has no associated data or the data will not be deposited. [Authors' comment: This is a theoretical research study, and is based upon analysis of the public experimental data, so no additional data is associated with this work.]

Open Access This article is licensed under a Creative Commons Attribution 4.0 International License, which permits use, sharing, adaptation, distribution and reproduction in any medium or format, as long as you give appropriate credit to the original author(s) and the source, provide a link to the Creative Commons licence, and indicate if changes were made. The images or other third party material in this article are included in the article's Creative Commons licence, unless indi- cated otherwise in a credit line to the material. If material is not included in the article's Creative Commons licence and your intended use is not permitted by statutory regulation or exceeds the permitted use, you will need to obtain permission directly from the copyright holder. To view a copy of this licence, visit http://creativecomm ons.org/licenses/by/4.0/.

Funded by $\mathrm{SCOAP}^{3}$.

\section{References}

1. D. Boer, M. Diehl, R. Milner, R. Venugopalan, W. Vogelsang, D. Kaplan, H. Montgomery, S. Vigdor et al., arXiv:1108.1713 [nucl-th]

2. A. Accardi, J.L. Albacete, M. Anselmino, N. Armesto, E.C. Aschenauer, A. Bacchetta, D. Boer, W. Brooks et al., Eur. Phys. J. A 52(9), 268 (2016)

3. E.C. Aschenauer et al., Rep. Prog. Phys. 82(2), 024301 (2019)

4. J.L. Abelleira Fernandez et al., J. Phys. G 39, 075001 (2012)

5. P. Agostini et al., arXiv:2007.14491 [hep-ex]

6. X.D. Ji, J. Phys. G 24, 1181-1205 (1998)

7. K. Goeke, M.V. Polyakov, M. Vanderhaeghen, Prog. Part. Nucl. Phys. 47, 401-515 (2001)

8. X. Ji, Phys. Rev. Lett. 91, 062001 (2003)

9. M. Diehl, Phys. Rep. 388, 41-277 (2003)

10. A.V. Belitsky, X. d Ji, F. Yuan, Phys. Rev. D 69, 074014 (2004)

11. A.V. Belitsky, A.V. Radyushkin, Phys. Rep. 418, 1-387 (2005)

12. M. Diehl, Eur. Phys. J. A 52(6), 149 (2016)

13. Y. Hagiwara, Y. Hatta, T. Ueda, Phys. Rev. D 94(9), 094036 (2016)

14. Y. Hatta, B.W. Xiao, F. Yuan, Phys. Rev. Lett. 116(20), 202301 (2016)

15. T. Altinoluk, N. Armesto, G. Beuf, A.H. Rezaeian, Phys. Lett. B 758, 373-383 (2016)

16. H. Mäntysaari, N. Mueller, B. Schenke, Phys. Rev. D 99(7), 074004 (2019)

17. F. Salazar, B. Schenke, Phys. Rev. D 100(3), 034007 (2019)

18. Y. Hatta, N. Mueller, T. Ueda, F. Yuan, Phys. Lett. B 802, 135211 (2020)

19. D. Boer, C. Setyadi, Phys. Rev. D 104(7), 074006 (2021)

20. X.D. Ji, Phys. Rev. D 55, 7114-7125 (1997)

21. Y. Hatta, B.W. Xiao, F. Yuan, Phys. Rev. D 95(11), 114026 (2017)

22. H. Mäntysaari, K. Roy, F. Salazar, B. Schenke, Phys. Rev. D 103(9), $094026(2021)$

23. M. McDermott, R. Sandapen, G. Shaw, Eur. Phys. J. C 22, 655-666 (2002)

24. L. Favart, M.V.T. Machado, Eur. Phys. J. C 29, 365-371 (2003)

25. H. Kowalski, L. Motyka, G. Watt, Phys. Rev. D 74, 074016 (2006)

26. M.V.T. Machado, Eur. Phys. J. C 59, 769-776 (2009)

27. E.C. Aschenauer, S. Fazio, K. Kumericki, D. Mueller, JHEP 09, 093 (2013)

28. V.P. Gonçalves, D.S. Pires, Phys. Rev. C 91(5), 055207 (2015)

29. F. Gelis, E. Iancu, J. Jalilian-Marian, R. Venugopalan, Ann. Rev. Nucl. Part. Sci. 60, 463 (2010)

30. H. Weigert, Prog. Part. Nucl. Phys. 55, 461 (2005)

31. J. Jalilian-Marian, Y.V. Kovchegov, Prog. Part. Nucl. Phys. 56, 104 (2006)

32. J. Jalilian-Marian, A. Kovner, L. McLerran, H. Weigert, Phys. Rev. D 55, 5414 (1997)

33. J. Jalilian-Marian, A. Kovner, and H. Weigert, Phys. Rev. D 59, 014014 (1999). ibid.59, 014015 (1999). ibid.59, 034007 (1999)

34. A. Kovner, J. Guilherme Milhano, H. Weigert, Phys. Rev. D 62, 114005 (2000)

35. H. Weigert, Nucl. Phys. A 703, 823 (2002)

36. E. Iancu, A. Leonidov, L. McLerran, Nucl. Phys. A 692, 583 (2001) 
37. E. Ferreiro, E. Iancu, A. Leonidov, L. McLerran, Nucl. Phys. A 701, 489 (2002)

38. A.H. Rezaeian, M. Siddikov, M. VandeKlundert, R. Venugopalan, Phys. Rev. D 87, 034002 (2013)

39. H. Kowalski, D. Teaney, Phys. Rev. D 68, 114005 (2003)

40. A.H. Rezaeian, I. Schmidt, Phys. Rev. D 88, 074016 (2013)

41. I.I. Balitsky, Phys. Rev. Lett. 81, 2024 (1998)

42. I.I. Balitsky, Phys. Lett. B 518, 235 (2001)

43. I.I. Balitsky, A.V. Belitsky, Nucl. Phys. B 629, 290 (2002)

44. Y.V. Kovchegov, Phys. Rev. D 60, 034008 (1999)

45. Y.V. Kovchegov, Phys. Rev. D 61, 074018 (2000)

46. R.J. Glauber, in Lecture in Theoretical Physics, ed. by W.E. Brittin, L.G. Duham, vol. 1 (Interscience, New York, 1959)

47. V.N. Gribov, Sov. Phys. JETP 29, 483 (1969)

48. V.N. Gribov, Sov. Phys. JETP 30, 709 (1970)

49. A.H. Mueller, Nucl. Phys. B 335, 115 (1990)

50. N. Armesto, Eur. Phys. J. C 26, 35 (2013)

51. H. Kowalski, T. Lappi, R. Venugopalan, Phys. Rev. Lett. 100, 022303 (2008)

52. E.R. Cazaroto, F. Carvalho, V.P. Goncalves, F.S. Navarra, Phys. Lett. B 671, 233 (2009)

53. H. Kowalski, T. Lappi, C. Marquet, R. Venugopalan, Phys. Rev. C 78, 045201 (2008)

54. J. Cepila, J.G. Contreras, M. Matas, Phys. Rev. D 99(5), 051502 (2019)
55. D. Bendova, J. Cepila, J.G. Contreras, M. Matas, Phys. Rev. D 100(5), 054015 (2019)

56. J. Cepila, J.G. Contreras, M. Matas, Phys. Rev. C 102(4), 044318 (2020)

57. J.L. Albacete, Nucl. Phys. A 957, 71-84 (2017)

58. E. Iancu, J.D. Madrigal, A.H. Mueller, G. Soyez, D.N. Triantafyllopoulos, Phys. Lett. B 750, 643-652 (2015)

59. B. Ducloue, E. Iancu, G. Soyez, D.N. Triantafyllopoulos, Phys. Lett. B 803, 135305 (2020)

60. K.J. Golec-Biernat, A.M. Stasto, Nucl. Phys. B 668, 345-363 (2003)

61. K.J. Eskola, P. Paakkinen, H. Paukkunen, C.A. Salgado, Eur. Phys. J. C 77(3), 163 (2017)

62. H. Mäntysaari, P. Zurita, Phys. Rev. D 98, 036002 (2018)

63. K. Golec-Biernat, M. Wusthoff, Phys. Rev. D 59, 014017 (1999)

64. K. Golec-Biernat, M. Wusthoff, Phys. Rev. D D60, 114023 (1999)

65. A.G. Shuvaev, K.J. Golec-Biernat, A.D. Martin, M.G. Ryskin, Phys. Rev. D 60, 014015 (1999)

66. F.D. Aaron et al. [H1], Phys. Lett. B 681, 391-399 (2009)

67. D. Bendova, J. Cepila, J.G. Contreras, V.P. Gonçalves, M. Matas, Eur. Phys. J. C 81(3), 211 (2021) 